\title{
OPEN Distinctive effects of executive dysfunction and loss of learning/ memory abilities on resting-state brain activity
}

Hideyuki Hoshi ${ }^{1}$, Yoko Hirata ${ }^{2}$, Momoko Kobayashi $^{3}$, Yuki Sakamoto ${ }^{3}$, Keisuke Fukasawa ${ }^{4}$ Sayuri Ichikawa ${ }^{4}$, Jesús Poza ${ }^{5,6,7}$, Víctor Rodríguez-González ${ }^{5,6}$, Carlos Gómez ${ }^{5,6}$ \& Yoshihito Shigihara ${ }^{1,3 凶}$

Dementia is a syndrome characterised by cognitive impairments, with a loss of learning/memory abilities at the earlier stages and executive dysfunction at the later stages. However, recent studies have suggested that impairments in both learning/memory abilities and executive functioning might co-exist. Cognitive impairments have been primarily evaluated using neuropsychological assessments, such as the Mini-Mental State Examination (MMSE). Recently, neuroimaging techniques such as magnetoencephalography (MEG), which assess changes in resting-state brain activity, have also been used as biomarkers for cognitive impairment. However, it is unclear whether these changes reflect dysfunction in executive function as well as learning and memory. In this study, parameters from the MEG for brain activity, MMSE for learning/memory, and Frontal Assessment Battery (FAB) for executive function were compared within 207 individuals. Three MEG parameters were used as representatives of resting-state brain activity: median frequency, individual alpha frequency, and Shannon's spectral entropy. Regression analysis showed that median frequency was predicted by both the MMSE and FAB scores, while individual alpha frequency and Shannon's spectral entropy were predicted by MMSE and FAB scores, respectively. Our results indicate that MEG spectral parameters reflect both learning/memory and executive functions, supporting the utility of MEG as a biomarker of cognitive impairment.

Dementia is a syndrome characterised by progressive deterioration of cognitive functioning due to various brain pathologies. The most common form of dementia is Alzheimer's disease (AD), accounting for approximately $60-80 \%$ of all dementia cases ${ }^{1}$. Its effects include impaired learning/memory, language, problem-solving, attention, and behaviour. The loss of learning/memory abilities is often the chief complaint of patients with earlystage dementia ${ }^{2}$. These functions are served by the temporal cortex, where pathological change initially starts ${ }^{2}$. The Mini-Mental State Examination (MMSE), which is the most commonly used assessment tool for dementia screening ${ }^{3}$, primarily evaluates learning/memory performance ${ }^{4}$. Executive function is another sub-domain of cognitive function that includes attention, inhibition, working memory, interference control, and cognitive flexibility ${ }^{5}$, which is served by the prefrontal cortex ${ }^{6}$. It is believed that executive function is not affected until the later stages of $\mathrm{AD}^{2}$; however, recent studies have revealed that it can manifest in the earlier stages 7 .

Although learning/memory and executive functions interact with each other, they are independent to some extent $^{8}$ and are served by different brain regions. Hence, the tools used to measure both are also different: the MMSE for learning/memory ${ }^{4}$ and the Frontal Assessment Battery (FAB) for executive function ${ }^{9}$. The independence of these sub-domains of cognitive function is reflected by the fact that the MMSE is not sensitive to

${ }^{1}$ Precision Medicine Centre, Hokuto Hospital, Kisen-7-5 Inadacho, Obihiro, Hokkaido 080-0833, Japan. ${ }^{2}$ Department of Neurosurgery, Kumagaya General Hospital, Kumagaya 360-8567, Japan. ${ }^{3}$ Precision Medicine Centre, Kumagaya General Hospital, Kumagaya 360-8567, Japan. ${ }^{4}$ Clinical Laboratory, Kumagaya General Hospital, Kumagaya 360-8567, Japan. ${ }^{5}$ Biomedical Engineering Group, Higher Technical School of Telecommunications Engineering, University of Valladolid, 47011 Valladolid, Castilla y León, Spain. ${ }^{6}$ Centro de Investigación Biomédica en Red en Bioingeniería, Biomateriales Y Nanomedicina, (CIBER-BBN), 47011 Valladolid, Castilla y León, Spain. ${ }^{7}$ Instituto de Investigación en Matemáticas (IMUVA), University of Valladolid, 47011 Valladolid, Castilla y León, Spain. ${ }^{\varpi}$ email: y-shigihara@hokuto7.or.jp 


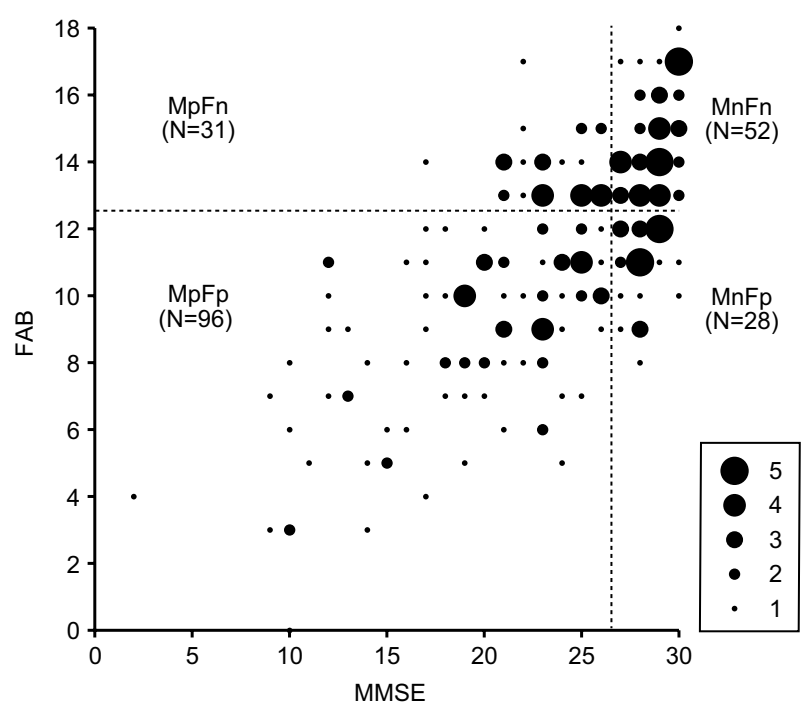

Figure 1. Relationship between Mini-Mental State Examination (MMSE) and Frontal Assessment Battery (FAB) scores. The sizes of the circles indicate the number of individuals who obtained a given score, and figures in the brackets represent the number of individuals who belong to each group. Broken lines indicate cut-off scores. Mn, MMSE-negative; Fn, FAB-negative; Mp, MMSE-positive; Fp, FAB-positive.

frontal-executive dysfunction ${ }^{10}$, and fails to detect mild cognitive impairment (MCI) in approximately a third of individuals ${ }^{11}$. Moreover, although both neuropsychological assessments are validated and easy to use in clinical settings, they have inherent limitations, such as skill dependence, subjectivity, ceiling effects, and practice effects.

To overcome these limitations, neuroimaging techniques such as electroencephalography (EEG) and magnetoencephalography (MEG) have come into use. EEG and MEG are able to evaluate resting-state brain activity in terms of neural oscillatory activity, whose changes are associated with cognitive impairment ${ }^{12-16}$. The changes are represented by (1) enhanced low-frequency oscillatory activity accompanied by attenuated high-frequency oscillatory activity, (2) slowing down of the alpha peak frequency, (3) less prominent alpha oscillations, and (4) loss of diversity of neural oscillatory components ${ }^{17-19}$. Previous studies have shown that these characteristics vary significantly between healthy individuals and individuals with MCI or $\mathrm{AD}^{19,20}$; hence, they can be considered potential biomarkers of cognitive impairment. However, previous studies have not evaluated the performance of these biomarkers in different sub-domains of cognitive impairment, which is crucial considering that dementia is defined by cognitive symptoms rather than a causative pathology. Therefore, in this study, we retrospectively analysed whether the resting-state brain activity acquired using MEG can assess learning/memory and executive functions-assessed using MMSE and FAB scores. Our analyses aimed to determine whether MEG biomarkers are sensitive to learning/memory and executive function.

\section{Results}

Descriptive statistics. Clinical data from 207 individuals were retrospectively analysed. The average MMSE and FAB scores were 23.4 \pm 5.6 (mean \pm standard deviation, SD) (ranging from 2 to 30 ) and $11.2 \pm 3.3$ $($ mean \pm SD) (ranging from 0 to 18), respectively. All individuals were divided into four groups according to their scores. Ninety-six individuals scored lower than the cut-off values for both MMSE (26 or below) and FAB (12 or below) (MMSE positive and FAB positive, MpFp), and 52 scored higher than those cut-offs (MMSE negative and FAB negative, MnFn). Thirty-one individuals scored lower than the MMSE cut-off but higher than the FAB cut-off (MpFn), and 28 scored higher than the MMSE cut-off but lower than the FAB cut-off (MnFp). This means that MMSE and FAB scores were mismatched for $59(31+28)$ individuals (see Fig. 1). The detailed descriptive statistics and group-level differences are described in Supplementary information (Descriptive Statistics).

Correlation analysis. Three MEG spectral parameters were introduced to quantify the properties of resting-state brain activity: median frequency (MF), individual alpha frequency (IAF), and Shannon's spectral entropy (SSE). They were compared with age and MMSE and FAB scores using bootstrap correlation analysis. Age was negatively correlated with both neuropsychological assessment scores (MMSE: $r=-0.258, p<0.001$ and FAB: $r=-0.361, p<0.001)$ and two MEG spectral parameters (MF: $r=-0.139, p=0.013$ and IAF: $r=-$ $0.150, p=0.015)$, except for SSE $(r=-0.106, p=0.059)$ (Table 1 and Fig. 2). Age showed greater correlations with the MMSE and FAB scores than with the MEG spectral parameters. All MMSE and FAB scores and MEG spectral parameters correlated positively with each other. The MMSE and FAB scores were correlated positively $(r=0.697, p<0.001)$.

Regression analyses. Directional relationships between MEG spectral parameters and neuropsychological assessments were studied using a linear mixed-effect model (LMEM), where biases from individuals' age 


\begin{tabular}{|c|c|c|c|c|c|c|c|c|c|c|}
\hline & \multicolumn{2}{|l|}{ AGE } & \multicolumn{2}{|c|}{ MMSE } & \multicolumn{2}{|l|}{ FAB } & \multicolumn{2}{|l|}{ MF } & \multicolumn{2}{|l|}{ IAF } \\
\hline & $r$ & $p$ (FDR) & $r$ & $p$ (FDR) & $r$ & $p$ (FDR) & $r$ & $p$ (FDR) & $r$ & $p$ (FDR) \\
\hline MMSE & -0.258 & $<0.001^{*}$ & & & & & & & & \\
\hline FAB & -0.361 & $<0.001^{*}$ & 0.697 & $<0.001^{*}$ & & & & & & \\
\hline MF & -0.139 & $0.013^{*}$ & 0.460 & $<0.001^{*}$ & 0.437 & $<0.001^{*}$ & & & & \\
\hline IAF & -0.150 & $0.015^{*}$ & 0.439 & $<0.001^{*}$ & 0.413 & $<0.001^{*}$ & 0.780 & $<0.001^{*}$ & & \\
\hline SSE & -0.106 & 0.059 & 0.221 & $0.001^{*}$ & 0.301 & $<0.001^{*}$ & 0.635 & $<0.001^{*}$ & 0.349 & $<0.001^{\star}$ \\
\hline
\end{tabular}

Table 1. Correlation matrix between age, neuropsychological assessment scores, and MEG spectral parameters. MMSE, Mini-Mental State Examination; FAB, Frontal Assessment Battery; MF, Median Frequency; IAF, Individual Alpha Frequency; SSE, Shannon's Spectral Entropy; $r$, averaged correlation coefficient across bootstrap iterations; $p$ (FDR), $p$-values of bootstrapping statistics with FDR correction. An asterisk $\left(^{*}\right)$ indicates statistically significant correlations after FDR correction.
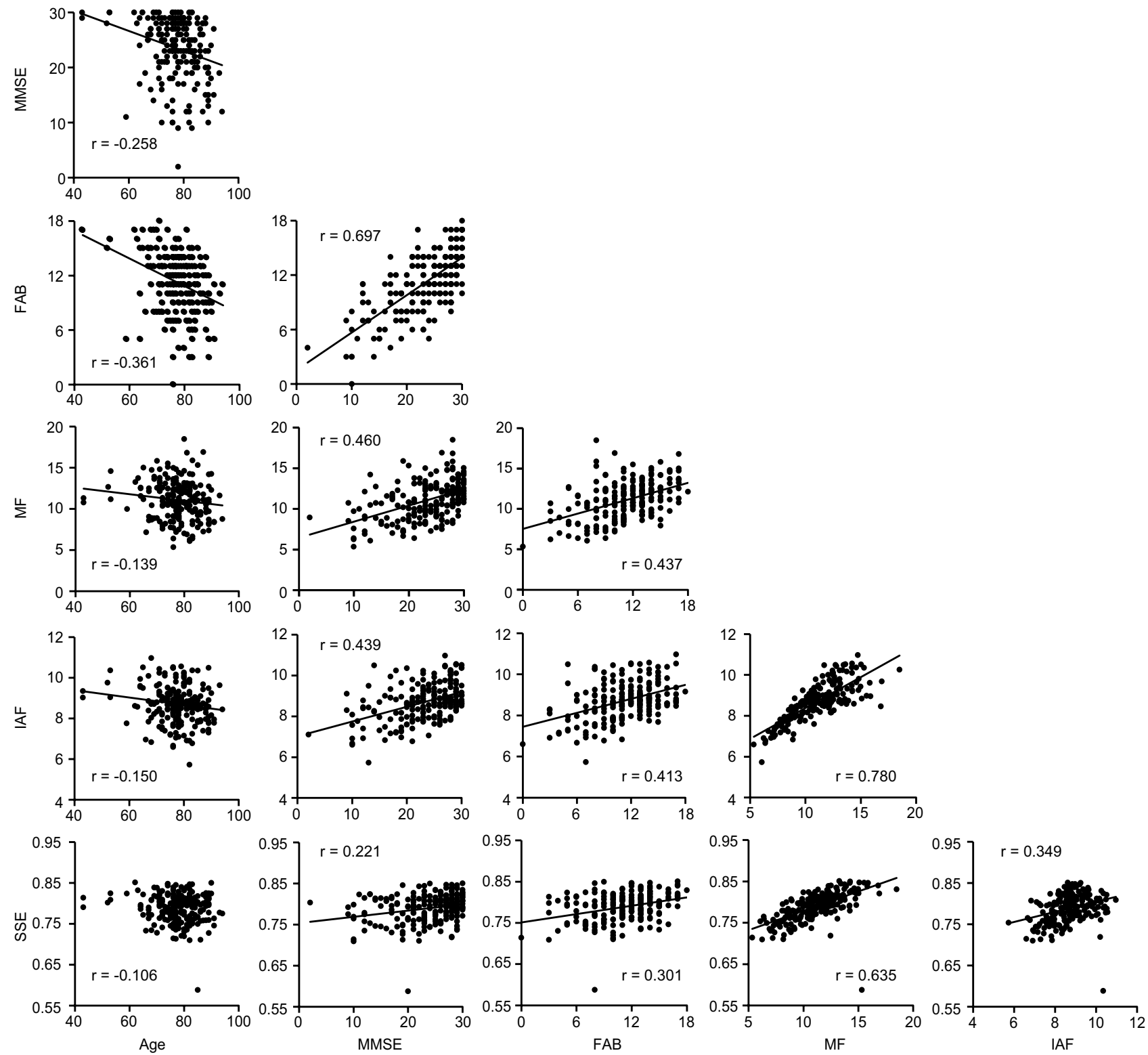

Figure 2. Correlations between age, neuropsychological assessment scores, and MEG spectral parameters. All pairs show significant correlations, except for age and SSE. Regression lines are added for significant correlations. MF and IAF are expressed in Hertz, and age in years. MMSE, Mini-Mental State Examination; FAB, Frontal Assessment Battery; MF, Median Frequency; IAF, Individual Alpha Frequency; SSE, Shannon's Spectral Entropy; r, averaged correlation coefficient across bootstrap iterations. 


\begin{tabular}{|c|c|c|c|c|c|c|c|c|c|c|c|c|}
\hline & \multicolumn{4}{|l|}{ MF } & \multicolumn{4}{|l|}{ IAF } & \multicolumn{4}{|l|}{ SSE } \\
\hline & $\beta$ & $S E$ & $t$ & $p$ (FDR) & $\beta$ & $S E$ & $t$ & $p$ (FDR) & $\beta$ & $S E$ & $t$ & $p$ (FDR) \\
\hline Intercept & -0.019 & 0.113 & -0.169 & 0.992 & -0.022 & 0.097 & -0.226 & 0.992 & -0.001 & 0.081 & -0.010 & 0.992 \\
\hline Age & 0.006 & 0.067 & 0.089 & 0.992 & -0.014 & 0.096 & -0.148 & 0.992 & -0.008 & 0.097 & -0.080 & 0.992 \\
\hline MMSE & 0.298 & 0.084 & 3.555 & $0.005^{*}$ & 0.291 & 0.085 & 3.421 & $0.005^{*}$ & 0.020 & 0.092 & 0.214 & 0.992 \\
\hline FAB & 0.214 & 0.087 & 2.446 & $0.046^{*}$ & 0.185 & \begin{tabular}{|l|}
0.089 \\
\end{tabular} & 2.094 & 0.090 & 0.282 & 0.095 & 2.955 & $0.014^{*}$ \\
\hline
\end{tabular}

Table 2. Results of LMEM examining the effects of age and neuropsychological assessment scores on MEG spectral parameters. MMSE, Mini-Mental State Examination; FAB, Frontal Assessment Battery; MF, Median Frequency; IAF, Individual Alpha Frequency; SSE, Shannon's Spectral Entropy; $\beta$ standardised estimated coefficient of the predictor; $t$ t $t$-values for testing the null hypothesis that the coefficients are equal to zero; $S E$, standard error of estimated coefficient; $p$ (FDR), $p$-values of t-test with FDR correction. An asterisk $\left(^{*}\right)$ indicates terms that significantly contributed to the model.

\section{Neuropsychological factors}

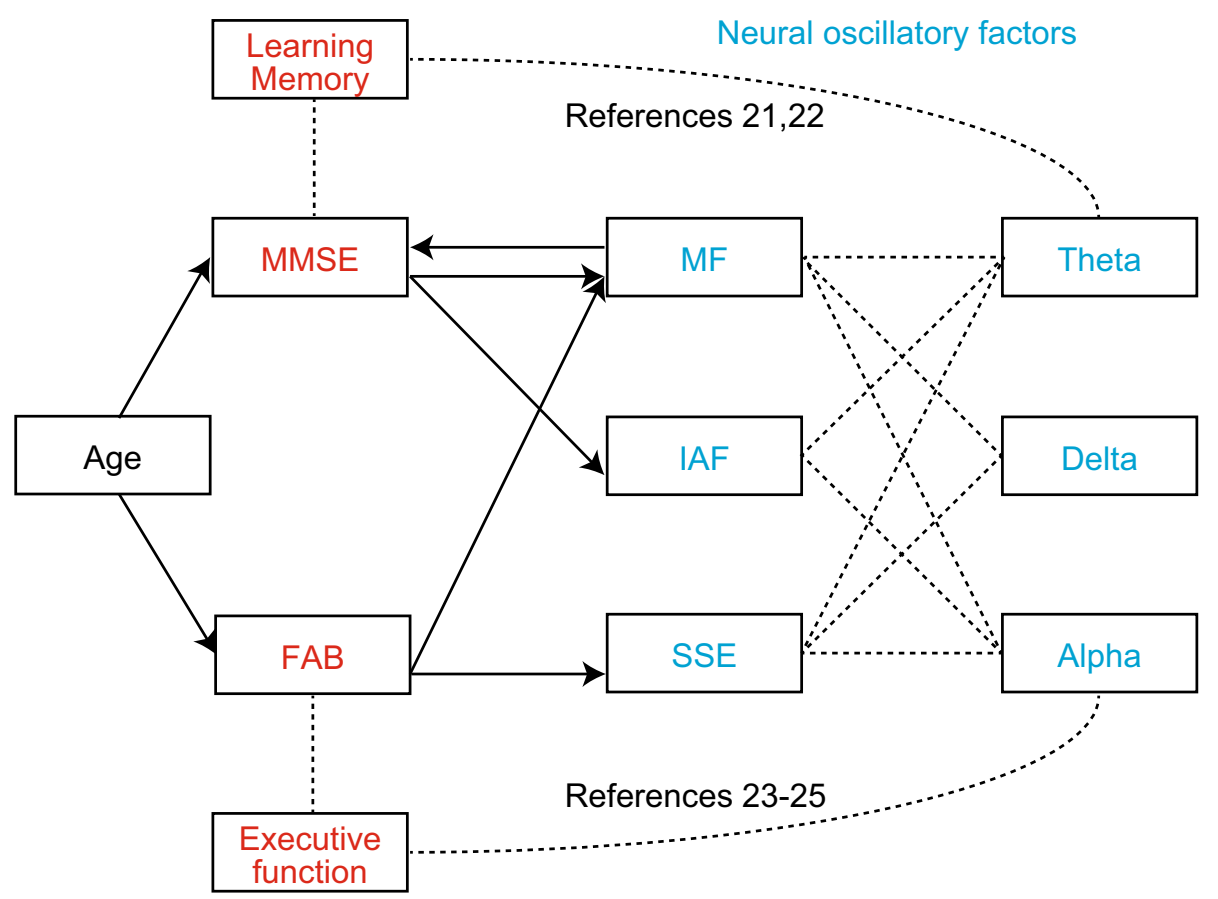

Figure 3. Associations between age, neuropsychological assessment scores, and MEG spectral parameters. Arrows indicate significant directional influence revealed by regression analysis. Dashed lines indicate associations described in previous studies ${ }^{21-25}$. MMSE, Mini-Mental State Examination; FAB, Frontal Assessment Battery; MF, Median Frequency; IAF, Individual Alpha Frequency; SSE, Shannon's Spectral Entropy.

and sex were considered as nuisance variables. The LMEM analysis was performed bi-directionally, with either MEG spectral parameters or neuropsychological assessments used as response variables. MF was predicted by both MMSE $(\beta=0.298, p=0.005)$ and FAB $(\beta=0.214, p=0.046)$ scores. IAF was predicted only by MMSE score $(\beta=0.291, p=0.005)$, but not FAB. SSE was predicted only by FAB scores $(\beta=0.282, p=0.014)$ (Table 2 and Fig. 3). MMSE scores were predicted by age $(\beta=-0.190, p=0.009)$ and $\mathrm{MF}(\beta=0.410, p=0.012)$, but not by IAF or SSE. FAB scores were predicted only by age $(\beta=-0.301, p<0.001)$, but not by any of the MEG spectral parameters (Table 3 and Fig. 3). Two additional analyses were performed to further examine these results. For the first analysis, we applied an analogous protocol on the frontal and temporal sensors of interest (SOIs) (instead of all 160 sensors) as executive function (FAB) and learning/memory (MMSE) depend on these areas ${ }^{2,6}$. The results are described in Supplementary information (Regression analysis with MEG sensors of interest). For the second analysis, we considered only the healthy individuals' dataset, as cognitive decline due to healthy ageing and pathological change could lead to different relationships between MEG spectral parameters and neuropsychological assesment scores; hence, we used the dataset of 34 individuals with healthy ageing (a subset of the present dataset) in the control analysis. The results are shown in the Supplementary information (Regression analysis with individuals with healthy ageing). 


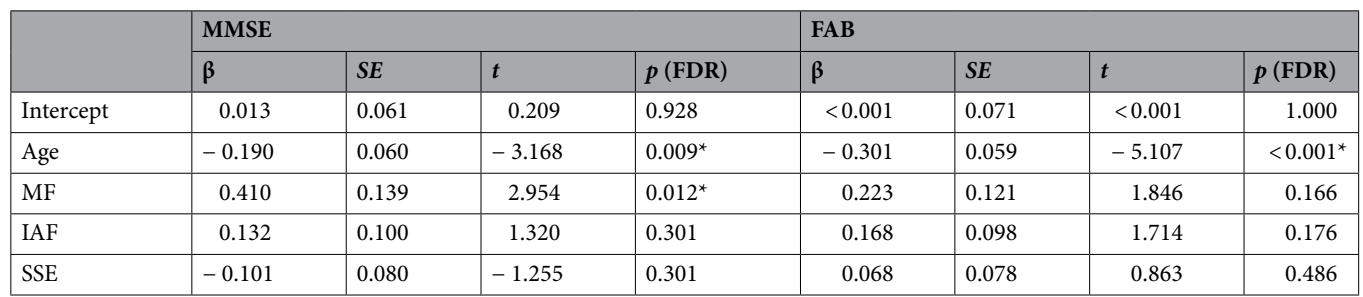

Table 3. Results of LMEM examining the effects of age and MEG spectral parameters on neuropsychological assessment scores. MMSE, Mini-Mental State Examination; FAB, Frontal Assessment Battery; MF, Median Frequency; IAF, Individual Alpha Frequency; SSE, Shannon's Spectral Entropy; $\beta$, standardised estimated coefficient of the predictor; $t$, t-values for testing the null hypothesis that the coefficients are equal to zero; $S E$, standard error of estimated coefficient; $p$ (FDR), $p$-values of t-test with FDR correction. An asterisk $\left({ }^{*}\right)$ indicates terms that significantly contributed to the model.

\section{Discussion}

This study showed that both sub-domains of cognitive functions-learning/memory and executive functionwere associated with MEG spectral parameters. MF was sensitive to both sub-domains, while IAF and SSE were only sensitive to learning/memory and executive function, respectively.

Dementia is a medical condition that is not defined by a causative pathology, but by symptoms that reflect deteriorating cognitive functions such as learning, memory, and executive function. It is believed that while learning/memory is affected in the earlier stages of $\mathrm{AD}$, executive function is not. A previous study reported that decline in episodic memory occurs 7 years before the diagnosis of dementia, while decline in executive function occurs 2-3 years before diagnosis at group-level ${ }^{26}$. The early onset of decline in memory is supported by the fact that loss of learning/memory ability is served by atrophy observed in the temporal lobe, which changes during the earlier stages of AD. However, recent studies have shown that a deficit in executive function can also occur during the early stages of $\mathrm{AD}^{7}$. Particularly, pathological changes in the prefrontal cortex can manifest early due to reduced white matter tract integrity rather than cortical atrophy ${ }^{27}$. This aligns with our findings of 28 individuals scoring lower than the FAB threshold, but not the MMSE threshold (MnFp). Moreover, these results imply that executive dysfunction can precede the dysfunction in learning/memory at the individual level. In contrast, 31 individuals were MpFn, implying that learning/memory (i.e. MMSE) and executive function (i.e. FAB) are independent to some extent, and dysfunctions in both sub-domains are not always simultaneous. Therefore, to effectively screen patients with cognitive impairment at an early stage, cognitive dysfunction should be evaluated at least in two axes: learning/memory and executive function. This raises the question of how these two sub-domains of cognition are associated with MEG biomarkers.

In the present study, we focused on three MEG spectral parameters (i.e. MF, IAF, and SSE) as potential biomarkers that are sensitive to cognitive impairment ${ }^{12-16}$. These parameters have been clinically used at Kumagaya General Hospital since 2019. Throughout the analysis, we also considered the influence of age, as it is a major risk factor for dementia ${ }^{28}$ and influences MEG spectral parameters across the lifespan ${ }^{29}$ (Table 1). Correlation analyses showed that both neuropsychological assessment scores correlated with age. Although MEG spectral parameters, except for SSE, also correlated with age, their correlation coefficients were smaller than those of neuropsychological assessments. Previous studies have shown that MEG signals can be affected by various biological conditions that are independent of age, such as fatigue ${ }^{30}$, endocrinological conditions $s^{31}$, and appetite a $^{32}$. Therefore, it is understandable that age is more weakly correlated with the MEG spectral parameters than with neuropsychological assesment scores. Correlation analyses also showed that neuropsychological assessment scores were associated with MEG spectral parameters, aligning with previous findings of associations between spontaneous neural oscillatory activity (i.e. resting-state brain activity) and cognitive impairment ${ }^{12-16}$.

Regression analyses showed that MF was predicted by both MMSE and FAB, while IAF and SSE were predicted by MMSE and FAB, respectively (Table 2 and Fig. 3). These results suggest that MF is sensitive to both sub-domains (learning/memory and executive function). Both MF and IAF represent the spectral power balance between low- and high-frequency neural oscillatory activity: MF reflects the power balance in a wide frequency range, while IAF mainly reflects the theta and alpha bands. They decrease due to increased slow neural oscillatory activity, such as delta and theta oscillatory activities. A previous study showed that an increase in theta oscillations was associated with decreased hippocampal volume ${ }^{33}$, whose major functions are learning/memory ${ }^{21}$. Other studies have shown that theta oscillations are associated with memory ${ }^{22,34}$. Thus, it is presumable that MF and IAF are associated with MMSE scores (i.e. learning/memory). Delta oscillations are associated with the cholinergic neuronal activity, whose single source is the nucleus basalis of Meynert in the basal forebrain, which projects both frontal and temporal cortices ${ }^{35}$, modulating both learning/memory ${ }^{36}$ and executive function ${ }^{37}$. As $\mathrm{AD}$ causes loss of cholinergic neurons in the nucleus basalis of Meynert ${ }^{38}$, and deafferentation of the cortex from cholinergic inputs increases cortical delta oscillations ${ }^{39}$, it is possible that changes in MF were associated with both learning/memory (i.e. temporal lobe) and executive functions (i.e. frontal lobe); although, IAF was not associated with executive functions.

The SSE is another spectral parameter that quantifies the irregularity of the normalised power spectral density (PSDn) of resting-state neural oscillatory activity. The SSE increases to 1.0 when oscillatory power is equally distributed across the whole frequency spectrum (i.e. white noise) and decreases to 0 when some specific frequencies 
are prominent. Previous studies have revealed that patients with AD have lower SSE values compared to cognitively healthy individuals ${ }^{40,41}$. Our findings support these studies by demonstrating that SSE was predicted by the FAB score (i.e. executive function; $\beta=0.282, p=0.014$ ), but not by the MMSE score (i.e. learning/memory; $\beta=0.020, p=0.992$ ), which is associated with theta oscillations ${ }^{22,34}$. This suggests that the association between SSE and FAB was affected by changes in higher frequency activities, such as alpha and beta. Alpha is not a single neural oscillatory activity; there are two or more alpha oscillations with distinctive cortical distributions and peak frequencies ${ }^{42}$. A previous study showed that non-pharmacological treatment improved cognitive function in patients with dementia, accompanied by a reduction in alpha oscillatory activity in the temporal $\operatorname{cortex}^{23}$. This suggests that larger temporal alpha oscillations are associated with lower values of SSE and severe cognitive dysfunction. Beta activity changes along with healthy ageing ${ }^{43}$; it attenuates in the early stage of $\mathrm{AD}^{44}$, and its power in the right frontal cortex is positively correlated with MMSE scores from patients with $\mathrm{MCI}^{45}$. A larger beta power predicted a more positive outcome of non-pharmacological treatments ${ }^{45}$, and non-pharmacological treatments enhance beta power in patients with dementia ${ }^{23}$. These results indicate that a reduction in beta oscillations can be associated with lower SSE values. Both alpha and beta oscillations are associated with attention ${ }^{24,25,46}$, working memory ${ }^{47,48}$, and inhibition ${ }^{25,47,49}$, which are all part of executive functions. We posit that changes in high-frequency oscillations (e.g. alpha and beta) mediate SSE and FAB.

Regression analyses also showed that the MMSE scores were predicted by age and MF, but not by IAF or SSE (Table 3 and Fig. 3), whereas the FAB scores were predicted only by age, but not by any of the MEG spectral parameters. The asymmetrical relationship between neuropsychological assessment scores and MEG spectral parameters can be explained by the role of age. Correlation analyses showed that age influenced neuropsychological assessment scores more than MEG spectral parameters (Table 1). It is assumed that age explained a large amount of variance in the LMEM predicting neuropsychological assesment scores, which left little room for MEG spectral parameters to contribute to the model (Table 3).

Analyses for all MEG sensors were replicated using MEG SOIs, namely MEG analysis using only frontal sensors (Fnt) or temporal sensors (Tmp), instead of using all 160 sensors, with results demonstrating minor differences [see Supplementary information (Regression analysis with MEG sensors of interest)]. When predicting MEG spectral parameters at SOIs using neuropsychological assesement scores, MF and IAF in both SOIs were predicted by MMSE scores, except TmpMF, whose significance did not meet the threshold after FDR correction ( $p=0.054$ ) (Supplementary Fig. S3 and Table S5). Further, MF and IAF in both SOIs were not predicted by FAB scores, although MF (for all MEG sensors) was predicted by both MMSE and FAB scores (Fig. 3). There are two possible explanations for this discrepancy. The first is the difference in signal to noise ratio. In terms of the standardised estimated coefficient of the LMEM, although the association of FAB scores with MF and IAF (for all MEG sensors) were significant (MF: $\beta=0.214$; IAF: $\beta=0.185$ ), the association of MMSE scores with the same was stronger (MF: $\beta=0.298$; IAF: $\beta=0.291$ ). A reduced number of sensors averaged within frontal (35) and temporal (42) SOIs failed to find statistically significant relationships. The second is the contribution of the other 83 MEG sensors, which are neither in Fnt nor Tmp SOIs. If these 83 sensors had any relationship with FAB scores, it would explain that their rejection in the SOI analysis weakens the contributions of FAB scores to the MEG spectral parameters.

Considering the other direction of causality, namely, predicting neuropsychological assesement scores using MEG spectral parameters, frontal and temporal sensors behaved differently (Supplementary Fig. S3 and Table S6). Although age influenced both MMSE and FAB scores in all MEG sensor analyses ("Regression analyses" section, Fig. 3, and Table 3) and FAB in SOI analysis, MMSE was not predicted by age in the SOI analysis (Supplementary Fig. S3 and Table S6). This is consistent with a previous review describing that cognitive impairments associated with the temporal lobe arise from the pathology of $\mathrm{AD}$, while impairments associated with the frontal lobe occur during healthy ageing ${ }^{50}$. The former contributes to changes in MMSE (i.e. learning/memory), while the latter contributes to changes in FAB (i.e. executive function). The difference in the contribution of age between the two regions led to this discrepancy. The MMSE score was only predicted by TmpSSE, but not by age or the rest of the MEG spectral parameters (Supplementary Fig. S3). The SSE reflects changes in the neural oscillatory activity at any frequency. Temporal delta, theta, alpha, and beta oscillations are associated with MMSE scores ${ }^{23,51}$. Hence, it is reasonable to conclude that TmpSSE influenced the MMSE score. Taken together, the relationships between MMSE scores (i.e. learning/memory) and MF/IAF were evident, while the FAB score (i.e. executive function) was associated only with SSE.

Regression analysis for individuals with healthy ageing [see Supplementary information (Regression analysis with individuals with healthy ageing)] did not show significant associations between MEG spectral parameters and neuropsychological assessments, which was evident for the whole database ("Regression analyses" section and Tables 2 and 3). The discrepancy can be interpreted in three ways. First, the significant associations were only limited to the pathological conditions. The neuropsychological assessment scores declined for two reasons: healthy ageing ${ }^{52,53}$ and pathological backgrounds, such as Alzheimer's disease ${ }^{54}$. When both sources of variability co-exist (using all 207 individuals), the relationships between MEG parameters and neuropsychological assessment scores were significant; however, when the source of the variability was limited to healthy ageing alone, the relationships did not survive. Previous studies show that resting-state brain activity is sensitive to pathological changes $^{55,56}$. These indicate that MEG parameters are mainly sensitive to a decline due to the pathological changes rather than healthy ageing. Second, the sample size of the dataset was reduced from 207 in the main analysis to 34 in the control analyses. The reduction in the number of examined individuals may have weakened the detection power of the group-level analysis, leading to missed associations between parameters. Third, the ceiling effect of the neuropsychological parameters ${ }^{57}$ reduced the variability of the dataset, which impacted their associations to MEG spectral parameters. Even under the limited conditions, FAB was significantly predicted by age. This result supports the findings of a previous study ${ }^{58}$, which posited that healthy ageing influences cognitive decline as well as the pathological changes. 
This study has some limitations. First, the MMSE is not a neuropsychological assessment used to evaluate learning/memory function selectively. However, this did not affect our conclusions because we employed the MMSE as it is not sensitive to executive dysfunction ${ }^{10}$, while the FAB is. The results demonstrated that MMSE and FAB scores mismatched for 59 individuals (Fig. 1), supporting the assumption that MMSE and FAB reflect different sub-domains of cognitive performance, albeit with some potential overlap.

Second, we used three MEG spectral parameters without considering the cortical sources of MEG signals (i.e. the analyses were performed at the sensor-level but not at the source-level) because we validated their performance as potential biomarkers in the same way as conducted in clinical practice. Small computational resources (less than $10 \mathrm{~min}$ ) and simplicity are essential features of clinical biomarkers, making sensor-level analysis outweigh source-level analysis. In addition, our previous study also supports the consistency of the chosen spectral parameters when they are computed at the source- or sensor-level ${ }^{59}$.

Third, this study used clinical records from individuals who had visited the outpatient department for dementia or received medical health check services. Few patients with severe dementia were included; thus, these findings might not reflect a wide range of pathological mechanisms pertinent to dementia. However, the goal of this study was to reveal the sensitivity of potential biomarkers to the two sub-domains of cognitive impairment for screening purposes. Hence, this limitation does not matter in practice.

Fourth, we did not examine classification performance on clinical categories (healthy ageing, MCI, and dementia) using MEG spectral parameters. We acknowledge that the MEG spectral parameters and neuropsychological assesment scores (i.e. MMSE and FAB) show no major differences between the clinical categories (between individuals with healthy ageing and MCI)(Supplementary Fig. S1). This indicates that the MEG spectral parameters have a closer relationship with neuropsychological assesment scores than clinical diagnoses. Notably, the relationship between neuropsychological assesment scores and clinical diagnosis is not that simple ${ }^{60}$ : an individual with an MMSE score of 30/30 can be diagnosed with dementia ${ }^{61}$. The diagnosis cannot be made using only neuropsychological assesment scores; the MEG spectral parameters are similar. We will address this topic with additional MEG spectral parameters to obtain a comprehensive characterisation of brain dynamics as the next step of our study series.

In conclusion, MEG spectral parameters reflect cognitive impairments not only in the learning/memory domain but also in executive function, which is sometimes affected in the earlier stages of cognitive impairment than learning/memory. Furthermore, this study also provides evidence for the application of MEG spectral parameters as clinical biomarkers of cognitive impairment, such as MCI and dementia.

\section{Methods}

Participants. We obtained neuropsychological (i.e. cognitive) assessment scores and resting-state MEG data retrospectively from 207 individuals ( 119 women; mean age \pm SD: $77.5 \pm 8.0$ years old, range $43-94$ years). They had visited the outpatient department of dementia or received medical health check services and underwent both MMSE/FAB assessments and MEG scans at the Kumagaya General Hospital between 1 April 2019 and 4 August 2021. All of them consented to their data being used for research. Regarding the clinical category, 34 were cognitively healthy individuals, 53 suffered from mild cognitive impairment, and 120 were diagnosed with dementia. Diagnoses were made by a neurosurgeon who was a clinical instructor at the Japanese Society of Dementia Research. The study was approved by the ethics committee of Kumagaya General Hospital (\#25 and \#40). All methods were performed in accordance with the relevant guidelines and regulations in Japan. All individuals provided written informed consent for participation in this study if they were cognitively healthy. If not, their caregivers provided consent on their behalf.

Neuropsychological assessments. Cognitive performance was assessed using the Japanese versions of the MMSE and FAB, administrated by clinical psychologists as a part of clinical practice. MMSE and FAB are scored on a scale of $0-30$ and $0-18$, respectively; lower scores indicate more severe cognitive impairment for both. Here, we defined participants who scored lower than the cut-off of the MMSE (i.e. 26 or below) ${ }^{53}$ as MMSE-positive (Mp) and participants who scored lower than the cut-off of the FAB (i.e. 12 or below) ${ }^{62}$ as FABpositive (Fp); otherwise, participants were grouped as MMSE-negative (Mn) and FAB-negative (Fn).

MEG scanning. Five minutes of resting-state brain activity (i.e. spontaneous neural oscillatory activity) was recorded using a 160-channel whole-head type magnetoencephalography system (RICOH160-1; RICOH, Tokyo, Japan) in a magnetically shielded room at Kumagaya General Hospital as a part of clinical practice. During the scan, participants were asked to remain calm in the supine position with their eyes closed. The sampling frequency was $2000 \mathrm{~Hz}$ with $500 \mathrm{~Hz}$ low-pass filtering during the recording.

MEG analysis. We applied the same sensor-level analysis protocol used in our previous study ${ }^{45}$ during daily clinical practice at Kumagaya General Hospital. MEG data are sometimes contaminated by artefacts such as signal fluctuation caused by dental works and can deteriorate the quality of analysis. Before starting the detailed analyses, artefacts were manually removed using principal component analysis, if necessary, by experienced clinicians or clinical laboratory technicians. The technique was applied for 41 out of 207 MEG datasets as a part of the clinical practice. The number of removed components were adjusted for each dataset and limited to as few as possible. It was conducted using an analysis software provided by the MEG manufacturer ${ }^{31,56}$, which is authorised for clinical use by the Ministry of Health, Labour, and Welfare of Japan (equivalent to FDA approval). A $50-\mathrm{Hz}$ band-stop filter was applied to remove the power line noise. After removing the artefacts, all MEG analyses were performed offline using MATLAB (MathWorks, MA, USA). Three spectral parameters were calculated to summarise the different properties of spontaneous neural oscillations: MF, IAF, and SSE ${ }^{41}$. Their definitions 
and details can be found in our previous study ${ }^{19}$. The first parameter, MF, quantifies the frequency at which the spectral power is balanced between low and high frequencies. The frequency divides PSDn into two equal halves between 1 and $70 \mathrm{~Hz}$. The second parameter, IAF, represents the dominant frequency corresponding to the peak of the PSDn in the alpha band (i.e. the dominant alpha oscillation). It is defined similarly to MF, but adjusting the frequency range between 4 and $15 \mathrm{~Hz}$ (i.e. extended alpha band) to obtain a robust estimator of the dominant alpha oscillations ${ }^{19}$. The last parameter, SSE, is defined by applying the definition of the normalised Shannon entropy to the PSDn, which can be assimilated as a probability density function ${ }^{19}$ :

$$
\mathrm{SSE}=-\frac{1}{\log (N)} \cdot \sum_{f=1 \mathrm{~Hz}}^{70 \mathrm{~Hz}} \operatorname{PSDn}(f) \log [\operatorname{PSDn}(f)]
$$

where $N$ is the number of frequency bins of the PSDn. SSE represents an irregularity measure closely related to the concept of order in information theory, which quantifies the homogeneity in the distribution of the oscillatory components of the PSDn. Sensor- and epoch-wise MF, IAF, and SSE were computed, then they were averaged across all sensors and epochs. They were referred to without any prefixes such as MF, IAF, and SSE, respectively. Additionally, to investigate the potential effects of the spatial location of sensors, the parameters were averaged within two groups of SOIs instead of whole sensors: frontal (Fnt) and temporal (Tmp) sensors (see Supplementary Fig. S2). The SOI data were used only as supplementary for LMEM analysis.

Statistical analysis. For clinical verification of data quality and enhancing the comprehensibility of the dataset, descriptive statistics were performed and basic group-level differences were examined [see Supplementary information (Descriptive Statistics)]. Next, correlation analyses were performed using a non-parametric bootstrapping approach to examine the relationships between the MEG spectral parameters and neuropsychological assessments. Bootstrapping statistics have methodological advantages over classical statistical inference (e.g. the Gaussian assumption ${ }^{63}$ ). Correlations were examined among each pair of variables (MF, IAF, SSE, MMSE, and FAB) and participants' ages. For each pair, Pearson's coefficient was calculated by resampling with replacement data across all individuals 20,000 times. The percentage of the resampled coefficients, when larger or smaller than 0 (the smaller value), was taken as the significance level ( $p$-value). We reported the grand average of the correlation coefficient $(r)$ across bootstrap iterations and $p$-values.

Next, the directional relationships between the MEG spectral parameters and neuropsychological assessments were studied using LMEM. The LMEM analysis was performed bidirectionally; contributions of MEG spectral parameters (predictor variables) on neuropsychological assessments (response variables) were studied, and vice versa. For each LMEM, the response variable was subjected to the regression model with three or two fixed covariates (MF, IAF, and SSE when taking neuropsychological scores as response variables; and MMSE and FAB when taking MEG parameters) with an additional fixed covariate: age. To consider sex differences in the model, a random intercept and random slopes (for all fixed predictors) were entered into the model for each sex. The model was estimated using the maximum-likelihood method. Estimated coefficients of fixed predictors were subjected to a $t$-test to check for the null hypothesis that the coefficients would be equal to zero.

As the analyses were exploratory and generated matrices (Tables 1,2,3) where each of the statistical values were tested against our null hypothesis (that the coefficients would be equal to zero), this series of results was at risk of an increasing Type-I error ${ }^{64}$. To manage this risk, we reported $p$-values controlled for the false discovery rate (FDR) using the Benjamini-Hochberg method $^{65}$. Significant values are marked with asterisks in all the tables. All statistical analyses were performed using the statistics and machine learning toolbox and multiple testing toolbox ${ }^{66}$ in the MATLAB software.

Ethics approval and consent to participate. The use of clinical data for the present study was approved by the ethics committee of Kumagaya General Hospital (\#25 and \#40). Additionally, written informed consent was obtained from the participants or their family members.

\section{Data availability}

All data generated or analysed during this study are available from Shigihara, Yoshihito (2021), 'MMSE and FAB study 2021', Mendeley Data, V1, https://doi.org/10.17632/b7bx8b52nx.1.

Received: 28 November 2021; Accepted: 11 February 2022

Published online: 02 March 2022

\section{References}

1. 2020 Alzheimer's disease facts and figures. Alzheimer's Dement. 16, 391-460 (2020).

2. Jahn, H. Memory loss in Alzheimer's disease. Dial. Clin. Neurosci. 15, 445-454 (2013).

3. Shigemori, K., Ohgi, S., Okuyama, E., Shimura, T. \& Schneider, E. The factorial structure of the mini mental state examination (MMSE) in Japanese dementia patients. BMC Geriatr. 10, 1-7 (2010).

4. Dinomais, M. et al. Anatomic correlation of the mini-mental state examination: A voxel-based morphometric study in older adults. PLoS ONE 11, e0162889 (2016).

5. Diamond, A. Executive functions. Annu. Rev. Psychol. 64, 135-168 (2013).

6. Gilbert, S. J. \& Burgess, P. W. Executive function. Curr. Biol. 18, R110-R114 (2008).

7. Guarino, A. et al. Executive functions in Alzheimer disease: A systematic review. Front. Aging Neurosci. 10, 437 (2019).

8. Han, M., Kim, D. Y., Leigh, J. H. \& Kim, M. W. Value of the frontal assessment battery tool for assessing the frontal lobe function in stroke patients. Ann. Rehabil. Med. 44, 261-272 (2020).

9. Dubois, B., Slachevsky, A., Litvan, I. \& Pillon, B. The FAB: A frontal assessment battery at bedside. Neurology 55, 1621-1626 (2000). 
10. Bak, T. H. \& Mioshi, E. A cognitive bedside assessment beyond the MMSE: The Addenbrooke's Cognitive Examination. Pract. Neurol. 7, 245-249 (2007).

11. Mitchell, A. J. A meta-analysis of the accuracy of the mini-mental state examination in the detection of dementia and mild cognitive impairment. J. Psychiatr. Res. 43, 411-431 (2009).

12. Fernández, A. et al. Focal temporoparietal slow activity in Alzheimer's disease revealed by magnetoencephalography. Biol. Psychiatry 52, 764-770 (2002).

13. Fernández, A. et al. Correlations of hippocampal atrophy and focal low-frequency magnetic activity in Alzheimer disease: Volumetric MR imaging-magnetoencephalographic study. Am. J. Neuroradiol. 24, 481-487 (2003).

14. Stam, C. J. Use of magnetoencephalography (MEG) to study functional brain networks in neurodegenerative disorders. J. Neurol. Sci. 289, 128-134 (2010).

15. Al-Qazzaz, N. K. et al. Role of EEG as biomarker in the early detection and classification of dementia. Sci. World J. 2014, 906038 (2014).

16. López-Sanz, D., Serrano, N. \& Maestú, F. The role of magnetoencephalography in the early stages of Alzheimer's disease. Front. Neurosci. 12, 572 (2018).

17. Fernández, A. et al. MEG delta mapping along the healthy aging-Alzheimer's disease continuum: Diagnostic implications. J. Alzheimers Dis. 35, 495-507 (2013).

18. López, M. E. et al. MEG spectral analysis in subtypes of mild cognitive impairment. Age 36, 9624 (2014).

19. Poza, J., Hornero, R., Abásolo, D., Fernández, A. \& García, M. Extraction of spectral based measures from MEG background oscillations in Alzheimer's disease. Med. Eng. Phys. 29, 1073-1083 (2007).

20. Poza, J. et al. Analysis of neural dynamics in mild cognitive impairment and Alzheimer's disease using wavelet turbulence. J. Neural Eng. 11, 026010 (2014).

21. Anand, K. S. \& Dhikav, V. Hippocampus in health and disease: An overview. Ann. Indian Acad. Neurol. 15, 239-246 (2012).

22. Klimesch, W. EEG alpha and theta oscillations reflect cognitive and memory performance: A review and analysis. Brain Res. Brain Res. Rev. 29, 169-195 (1999).

23. Shigihara, Y., Hoshi, H., Shinada, K., Okada, T. \& Kamada, H. Non-pharmacological treatment changes brain activity in patients with Dementia. Sci. Rep. 10, 6744 (2020).

24. Foxe, J. J. \& Snyder, A. C. The role of alpha-band brain oscillations as a sensory suppression mechanism during selective attention. Front. Psychol. 2, 154 (2011).

25. Klimesch, W. Alpha-band oscillations, attention, and controlled access to stored information. Trends Cogn. Sci. 16, 606-617 (2012).

26. Grober, E. et al. Memory impairment, executive dysfunction, and intellectual decline in preclinical Alzheimer's disease. J. Int. Neuropsychol. Soc. 14, 266-278 (2008).

27. Reinvang, I., Grambaite, R. \& Espeseth, T. Executive dysfunction in MCI: Subtype or early symptom. Int. J. Alzheimer's Dis. 2012 (2012).

28. Daviglus, M. L. et al. National institutes of health state-of-the-science conference statement: Preventing Alzheimer disease and cognitive decline. Ann. Intern. Med. 153, 176-181 (2010).

29. Gómez, C., Pérez-Macías, M. J., Poza, J., Fernández, A. \& Hornero, R. Spectral changes in spontaneous MEG activity across the lifespan. J. Neural Eng. 10, 066006 (2013).

30. Shigihara, Y. et al. Two types of mental fatigue affect spontaneous oscillatory brain activities in different ways. Behav. Brain Funct. 9, 2 (2013)

31. Haraguchi, R. et al. The menstrual cycle alters resting-state cortical activity: A magnetoencephalography study. Front. Hum. Neurosci. 15, 411 (2021).

32. Nakamura, C. et al. Neural effects of acute stress on appetite: A magnetoencephalography study. PLoS ONE 15, e0228039 (2020).

33. Grunwald, M., Hensel, A., Wolf, H., Weiss, T. \& Gertz, H. J. Does the hippocampal atrophy correlate with the cortical theta power in elderly subjects with a range of cognitive impairment?. J. Clin. Neurophysiol. 24, 22-26 (2007).

34. Kragel, J. E. et al. Hippocampal theta coordinates memory processing during visual exploration. Elife 9, e52108 (2020).

35. Mesulam, M. M., Mufson, E. J., Levey, A. I. \& Wainer, B. H. Cholinergic innervation of cortex by the basal forebrain: Cytochemistry and cortical connections of the septal area, diagonal band nuclei, nucleus basalis (substantia innominata), and hypothalamus in the rhesus monkey. J. Comp. Neurol. 214, 170-197 (1983).

36. Maurer, S. V. \& Williams, C. L. The cholinergic system modulates memory and hippocampal plasticity via its interactions with non-neuronal cells. Front. Immunol. 8, 1489 (2017).

37. Logue, S. F. \& Gould, T. J. The neural and genetic basis of executive function: Attention, cognitive flexibility, and response inhibition. Pharmacol. Biochem. Behav. 123, 45-54 (2014).

38. Gratwicke, J. et al. The nucleus basalis of Meynert: A new target for deep brain stimulation in dementia?. Neurosci. Biobehav. Rev. 37, 2676-2688 (2013).

39. Lopes da Silva, F. Neural mechanisms underlying brain waves: From neural membranes to networks Electroencephalogr. Clin. Neurophysiol. 79, 81-93 (1991).

40. Gómez, C. \& Hornero, R. Entropy and complexity analyses in Alzheimer's disease: An MEG study. Open Biomed. Eng. J. 4, 223-235 (2010).

41. Poza, J., Hornero, R., Escudero, J., Fernández, A. \& Sánchez, C. I. Regional analysis of spontaneous MEG rhythms in patients with Alzheimer's disease using spectral entropies. Ann. Biomed. Eng. 36, 141-152 (2008).

42. Knyazeva, M. G., Barzegaran, E., Vildavski, V. Y. \& Demonet, J. F. Aging of human alpha rhythm. Neurobiol. Aging 69, 261-273 (2018).

43. Hoshi, H. \& Shigihara, Y. Age- and gender-specific characteristics of the resting-state brain activity: A magnetoencephalography study. Aging 12, 21613-21637 (2020).

44. Jeong, J. EEG dynamics in patients with Alzheimer's disease. Clin. Neurophysiol. 115, 1490-1505 (2004).

45. Shigihara, Y. et al. Predicting the outcome of non-pharmacological treatment for patients with dementia-related mild cognitive impairment. Aging 12, 24101-24116 (2020).

46. Gola, M., Magnuski, M., Szumska, I. \& Wróbel, A. EEG beta band activity is related to attention and attentional deficits in the visual performance of elderly subjects. Int. J. Psychophysiol. 89, 334-341 (2013).

47. Wianda, E. \& Ross, B. The roles of alpha oscillation in working memory retention. Brain Behav. 9, e01263 (2019).

48. Schmidt, R. et al. Beta oscillations in working memory, executive control of movement and thought, and sensorimotor function. J. Neurosci. 39, 8231-8238 (2019).

49. Tempel, T., Frings, C. \& Pastötter, B. EEG beta power increase indicates inhibition in motor memory. Int. J. Psychophysiol. 150, 92-99 (2020).

50. Buckner, R. L. Memory and executive function in aging and AD: Multiple factors that cause decline and reserve factors that compensate. Neuron 44, 195-208 (2004).

51. Fonseca, L. C., Tedrus, G. M. A. S., Prandi, L. R., Almeida, A. M. \& Furlanetto, D. S. Alzheimer's disease: Relationship between cognitive aspects and power and coherence EEG measures. Arq. Neuropsiquiatr. 69, 875-881 (2011).

52. Murman, D. L. The impact of age on cognition. Semin. Hear. 36, 111-121 (2015).

53. Boban, M. et al. The reliability and validity of the mini-mental state examination in the elderly Croatian population. Dement. Geriatr. Cogn. Disord. 33, 385-392 (2012). 
54. Budson, A. \& Solomon, P. Memory Loss, Alzheimer's Disease, and Dementia: E-Book: A Practical Guide for Clinicians (Elsevier, 2021).

55. Nakamura, A. et al. Electromagnetic signatures of the preclinical and prodromal stages of Alzheimer's disease. Brain 141, 1470-1485 (2018).

56. Matsumoto, T. et al. The association between carotid blood flow and resting-state brain activity in patients with cerebrovascular diseases. Sci. Rep. 11, 1-11 (2021).

57. Trzepacz, P. T., Hochstetler, H., Wang, S., Walker, B. \& Saykin, A. J. Relationship between the montreal cognitive assessment and mini-mental state examination for assessment of mild cognitive impairment in older adults. BMC Geriatr. 15, 1-9 (2015).

58. Coen, R. F. et al. The frontal assessment battery: Normative performance in a large sample of older community-dwelling hospital outpatient or general practitioner attenders. J. Geriatr. Psychiatry Neurol. 29, 338-343 (2016).

59. Rodríguez-González, V. et al. Consistency of local activation parameters at sensor- and source-level in neural signals. J. Neural Eng. 17, 56020 (2020).

60. Tombaugh, T. N. \& McIntyre, N. J. The mini-mental state examination: A comprehensive review. J. Am. Geriatr. Soc. 40, 922-935 (1992).

61. Shiroky, J. S., Schipper, H. M., Bergman, H. \& Chertkow, H. Can you have dementia with an MMSE score of 30?. Am. J. Alzheimers. Dis. Other Demen. 22, 406-415 (2007).

62. Slachevsky, A. et al. Frontal assessment battery and differential diagnosis of frontotemporal dementia and Alzheimer disease. Arch. Neurol. 61, 1104-1107 (2004).

63. Wasserman, S. \& Bockenholt, U. Bootstrapping: Applications to psychophysiology. Psychophysiology 26, 208-221 (1989).

64. Curran-Everett, D. Multiple comparisons: Philosophies and illustrations. Am. J. Physiol. Regul. Integr. Comp. Physiol. 279, R1-R8 (2000).

65. Benjamini, Y. \& Hochberg, Y. Controlling the false discovery rate: A practical and powerful approach to multiple testing. J. R. Stat. Soc. Ser. B 57, 289-300 (1995).

66. Martínez-Cagigal, V. Multiple Testing Toolbox (https://www.mathworks.com/matlabcentral/fileexchange/70604-multiple-testi ng-toolbox), MATLAB Central File Exchange. (2021).

\section{Acknowledgements}

We thank the participants of this study and would like to affirm our genuine respect for their contributions to the continued progress in the medical sciences. We sincerely thank Dr. Hajime Kamada (Chairperson, Hokuto Hospital) and Dr. Ikuo Hashimoto (Chairperson, Kumagaya General Hospital) for providing access to the respective facilities.

\section{Author contributions}

H.H. and Y.S. designed the study. Y.H. diagnosed. M.K. and Y.S. performed neuropsychological assessments. K.F. and S.I. operated the MEG and organised the clinical data. H.H. and Y.S. analysed the data and prepared the figures and tables. H.H., J.P., V.R.G., C.G., and Y.S. wrote the manuscript. All authors reviewed the manuscript.

\section{Funding}

This study was partially sponsored by RICOH Co., Ltd., by Ministerio de Ciencia e Innovación/Agencia Estatal de Investigación/10.13039/501100011033/' and ERDF A way of making Europe under the grant PGC2018-098214A-I00; and by 'CIBER en Bioingeniería, Biomateriales y Nanomedicina (CIBER-BBN)' through 'Instituto de Salud Carlos III' co-funded with ERDF funds. The sponsors had no role in the study concept/design, methods, data analysis, or preparation of the paper. Financial support was used for English editing and publication fees.

\section{Competing interests}

Dr. Yoshihito Shigihara is leading a joint research project with RICOH Co., Ltd. (manufacturer of magnetoencephalography equipment). Mr. Hideyuki Hoshi is now employed by RICOH Co., Ltd. Mr. V. RodríguezGonzález received a PIF-UVa grant from the University of Valladolid. Dr. Yoko Hirata, Miss Momoko Kobayashi, Miss Yuki Sakamoto, Mr. Keisuke Fukasawa, Miss Sayuri Ichikawa, Dr. Jesús Poza, and Dr. Carlos Gómez declare no potential conflicts of interest.

\section{Additional information}

Supplementary Information The online version contains supplementary material available at https://doi.org/ 10.1038/s41598-022-07202-7.

Correspondence and requests for materials should be addressed to Y.S.

Reprints and permissions information is available at www.nature.com/reprints.

Publisher's note Springer Nature remains neutral with regard to jurisdictional claims in published maps and institutional affiliations.

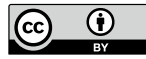

Open Access This article is licensed under a Creative Commons Attribution 4.0 International License, which permits use, sharing, adaptation, distribution and reproduction in any medium or format, as long as you give appropriate credit to the original author(s) and the source, provide a link to the Creative Commons licence, and indicate if changes were made. The images or other third party material in this article are included in the article's Creative Commons licence, unless indicated otherwise in a credit line to the material. If material is not included in the article's Creative Commons licence and your intended use is not permitted by statutory regulation or exceeds the permitted use, you will need to obtain permission directly from the copyright holder. To view a copy of this licence, visit http://creativecommons.org/licenses/by/4.0/.

(C) The Author(s) 2022 